\title{
Healthcare Encounters Start Relative to Reference Timepoint
}

National Cancer Institute

\section{Source}

National Cancer Institute. Healthcare Encounters Start Relative to Reference Timepoint. NCl Thesaurus. Code C124021.

An indication or description of the start of a healthcare encounter in relation to a specific point in time. 